\title{
CONVOLUTION MEASURE ALGEBRAS WITH GROUP MAXIMAL IDEAL SPACES $\left(^{1}\right)$
}

\author{
BY \\ JOSEPH L. TAYLOR
}

Let $G$ denote a locally compact abelian topological group (an 1.c.a. group) with dual group $G^{\wedge}$. We will denote by $M(G)$ the Banach algebra of bounded regular Borel measures on $G$ under convolution multiplication and by $L(G)$ the algebra of bounded measures absolutely continuous with respect to Haar measure on $G$ (for discussions of these Banach algebras cf. [1], [2], and [5]).

In this paper we shall be concerned with closed subalgebras $\mathfrak{M}$ of $M(G)$ with the following two properties:

(1) if $\mu \in \mathfrak{M}$ and $\nu$ is absolutely continuous with respect to $\mu$, then $\nu \in \mathfrak{M}$;

(2) the maximal ideal space of $\mathfrak{M}$ is $G^{\wedge}$, where the Gelfand transform $\mu^{\wedge}$ of $\mu \in \mathfrak{M}$ is given by $\mu^{\wedge}(\chi)=\int \bar{\chi} d \mu$ for $\chi \in G^{\wedge}$; i.e., the Gelfand transform coincides with the Fourier-Stieltjes transform on $\mathfrak{M}$.

Any closed subalgebra of $M(G)$ satisfying (1) will be called an $L$-subalgebra of $M(G)$. It is well known that $L(G)$ satisfies (1) and (2) (cf. [5, Chapter 1]). In Theorem 1 we show that any $L$-subalgebra $\mathfrak{M}$ of $M(G)$, with $L(G) \subset \mathfrak{M} \subset(L(G))^{1 / 2}$, also satisfies (2), where $(L(G))^{1 / 2}$ is the intersection of all maximal ideals of $M(G)$ containing $L(G)$. We conjecture that the converse is also true; i.e., if $\mathfrak{M}$ satisfies (1) and (2) then $L(G) \subset \mathfrak{M} \subset(L(G))^{1 / 2}$. In Theorem 2 we prove that this is true provided $G$ contains no copy of $R^{n}$ for $n>1$.

The problem arises in the following way: in [6] we define the concept of abstract convolution measure algebra and prove that any such algebra $\mathfrak{M}$, provided it is commutative and semisimple, may be represented as an $L$-subalgebra of $M(S)$, where $S$ is a compact topological semigroup called the structure semigroup of $\mathfrak{M}$. $M(G)$ and $L(G)$ are convolution measure algebras as is any $L$-subalgebra of the measure algebra on a semigroup. The map $\mu \rightarrow \mu_{S}$ which embeds $\mathfrak{M}$ in $M(S)$ is an isometry as well as an algebraic isomorphism and it preserves the order theoretic properties of $\mathfrak{M}$ as a space of measures. The maximal ideal space of $\mathfrak{M}$ may be identified as $S^{\wedge}$, the set of all semicharacters of $S$, where the Gelfand transform of $\mu \in \mathfrak{M}$ is given by $\mu^{\wedge}(f)=\int f d \mu_{S}$ for $f \in S^{\wedge}$ (a semicharacter of $S$ is a continuous homomorphism of $S$ into the unit disc which is not identically zero). Under pointwise multiplication $S^{\wedge}$ is a semigroup provided $\mathfrak{M}$ has an approximate identity.

Received by the editors March 14, 1966.

(1) Portions of these results were presented to the meeting of the Society held January 24-29, 1966, under the same title. This research was supported in part by the United States Army Research Office (Durham). 
Now, given this representation of $\mathfrak{M}$, it is natural to try to relate the algebraic structures of $S$ and $S^{\wedge}$ to the structure of $\mathfrak{M}$. In particular, if $S$ and $S^{\wedge}$ are groups, what can be said about $\mathfrak{M}$ ? More generally, if $G_{1}$ is a maximal group contained in $S$, then its dual group $G_{1}^{\wedge}$ is embedded in $S^{\wedge}$ (cf. [6, §3]) and, if $G_{1}$ is not a set of measure zero for $\mathfrak{M}$, then $G_{1}^{\wedge}$ is locally compact in the Gelfand topology of $S^{\wedge}$ and, with this topology, is the maximal ideal space of $\mathfrak{M}_{1}$, the algebra of measures in $\mathfrak{M}$ which are concentrated on $G_{1}$. It follows that $G_{1}^{\wedge}$, with the Gelfand topology (as opposed to the discrete topology it inherits from the compact group $G_{1}$ ), is the dual group $G^{\wedge}$ of some l.c.a. group $G$ (with Bohr compactification $G_{1}$ ) and that $\mathfrak{M}_{1}$ may be embedded as an $L$-subalgebra of $M(G)$. Thus $\mathfrak{M}_{1}$ is a subalgebra of $M(G)$ satisfying (1) and (2); i.e., by studying the relationship between maximal groups of $S$ and the structure of $\mathfrak{M}$ we are led to the problem posed in this paper.

Our problem may also be viewed as an attempt to extend results of Rieffel in [4]. Rieffel proves that if $A$ is a commutative semisimple Banach algebra which is Tauberian and in which every multiplicative linear functional is $L^{\prime}$-inducing, then $A$ is $L(G)$ for some 1.c.a. group $G$. The hypothesis that every multiplicative linear functional is $L^{\prime}$-inducing is equivalent to the existence of an 1.c.a. group $G$ such that $A$ is a closed subalgebra of $M(G)$ satisfying (1) and (2). The Tauberian hypothesis is extremely strong; it means that those elements of $A$ whose transforms have compact support on $G^{\wedge}$ are dense in $A$. If our conjecture is true, then without Rieffel's Tauberian hypothesis we may still conclude that $L(G) \subset A \subset(L(G))^{1 / 2}$. In Theorem 5.1 of [4] Rieffel mentions an example which shows that $L(G)$ and $(L(G))^{1 / 2}$ may be distinct.

We now proceed with our development of Theorems 1 and 2. If $B$ is any commutative Banach algebra and $I$ is a closed ideal of $B$, then $I^{1 / 2}$ denotes the intersection of all maximal ideals of $B$ which contain $I$. Under the natural map $x \rightarrow \xi_{x}$ from $B$ to $B / I, I^{1 / 2}$ is the inverse image of $(0)^{1 / 2}$; but $\xi_{x} \in(0)^{1 / 2}$ if and only if $\lim _{n}\left\|\xi_{x}^{n}\right\|^{1 / n}=0$ (cf. $[2,24 B]$ ). Hence, $x \in I^{1 / 2}$ if and only if $\lim _{n} \inf _{y \in I}\left\|x^{n}+y\right\|^{1 / n}=0$. In Lemma 1 we use this fact to obtain a measure theoretic characterization of the radical of an $L$-ideal, where an $L$-ideal $\mathfrak{R}$ of a convolution measure algebra $\mathfrak{M}$ is an ideal such that whenever $\mu \in \mathfrak{R}$ and $\nu \in \mathfrak{M}$ with $\nu$ absolutely continuous with respect to $\mu$, then $\nu \in \mathfrak{R}$.

If two measures $\mu$ and $\nu$ are mutually singular, then we write $\mu \perp \nu$. If $\mathfrak{R}_{1}$ and $\mathfrak{R}_{2}$ are sets of measures, then $\mathfrak{R}_{1} \perp \mathfrak{R}_{2}$ means $\mu \perp \nu$ for every $\mu \in \mathfrak{R}_{1}$ and $\nu \in \mathfrak{R}_{2}$. By $|\mu|$ we mean the measure defined by the total variation of $\mu$.

LEMMA 1. If $\mathfrak{R}$ is an L-ideal in a convolution measure algebra $\mathfrak{M}$, then $\mathfrak{R}^{1 / 2}$ is also an L-ideal and $\mu \in \mathfrak{N}^{1 / 2}$ if and only if $\mu \perp\left\{\nu \in \mathfrak{M}:|\nu|^{n} \perp \mathfrak{N}\right.$ for $\left.n=1,2, \ldots\right\}$.

Proof. Let $S$ be the structure semigroup of $\mathfrak{M}$ as in [6] and $\mu \rightarrow \mu_{S}$ the embedding of $\mathfrak{M}$ into $M(S)$. Let $A$ be the smallest closed subset of $S$ on which each $\mu_{S}$ for $\mu \in \mathfrak{N}$ is concentrated. A maximal regular ideal of $\mathfrak{M}$ containing $\mathfrak{R}$ corresponds to a semicharacter $f \in S^{\wedge}$ such that $\int f d \mu_{S}=0$ for each $\mu \in \mathfrak{N}$. Since $\mathfrak{N}$ is an $L$-ideal it 
follows that such an $f$ must be identically zero on $A$. Let $J$ be the set of all such semicharacters $f$ together with the identically zero function on $S$; then $J$ is closed under conjugation and pointwise multiplication. Let $A^{\prime}=\{s \in S: f(s)=0$ for all $f \in J\}$. Since $S^{\wedge}$ separates points in $S$ (cf. [6, Theorem 2.2]), $J$ separates points in $S \backslash A^{\prime}$ and, thus, the Stone-Weierstrass theorem implies that the closed linear span of $J$ in $C(S)$ consists of those continuous functions which vanish on $A^{\prime}$. Now $\mathfrak{N}^{1 / 2}$ consists of those measures $\mu$ in $\mathfrak{M}$ for which $\int f d \mu_{S}=0$ for all $f \in J$; i.e., those measures $\mu \in \mathfrak{M}$ for which $\mu_{S}$ is concentrated on $A^{\prime}$. It follows that $\mathfrak{R}^{1 / 2}$ is an $L$-ideal.

Since $\mathfrak{N}^{1 / 2}$ is an $L$-ideal, $\mu \in \mathfrak{N}^{1 / 2}$ if and only if $|\mu| \in \mathfrak{R}^{1 / 2}$. Therefore, it suffices to prove the second part of Lemma 1 for positive $\mu$. Let $\mu \in \mathfrak{M}, \mu \geqq 0$, and $\nu \in \mathfrak{M}$ such that $|\nu|^{n} \perp \mathfrak{N}$ for $n=1,2, \ldots$; then if $\mu$ and $\nu$ are not mutually singular, we may write $\mu=\mu_{1}+\mu_{2}$ where $\mu_{1}, \mu_{2} \geqq 0$ and $0 \neq \mu_{2} \leqq|\nu|$. Then $\left\|\omega+\mu^{n}\right\| \geqq\left\|\mu_{2}\right\|^{n}$ for every $\omega \in \mathfrak{R}$, since $\mu_{2}^{n} \leqq|\nu|^{n} \perp \mathfrak{N}$. Hence $\lim _{n} \inf _{\omega \in \mathfrak{N}}\left\|\omega+\mu^{n}\right\|^{1 / n} \geqq\left\|\mu_{2}\right\| \neq 0$, and $\mu \notin \mathfrak{R}^{1 / 2}$. Thus if $\mu \in \mathfrak{N}^{1 / 2}$ then $\mu \perp \nu$ whenever $\nu \in \mathfrak{M}$ and $|\nu|^{n} \perp \mathfrak{N}$ for $n=1,2, \ldots$

To see the converse, note that if $\mu \notin \mathfrak{R}^{1 / 2}$ then there is a semicharacter $f$ in the set $J$ described above for which $\int f d \mu_{S} \neq 0$. We may write $\mu=\omega+\nu$ where $\omega_{S}$ is concentrated on the set where $f$ is zero and $\nu_{S}$ is concentrated on the set where $f$ is nonzero. Since $f$ is multiplicative, $\nu_{S}^{n}$ is also concentrated on the set where $f$ is nonzero and, hence, $|\nu|^{n} \perp \mathfrak{N}$ for each $n$. This completes the proof, since $\mu$ and $\nu$ are not mutually singular.

THEOREM 1. If $\mathfrak{M}$ is an L-subalgebra of $M(G)$ and $L(G) \subset \mathfrak{M} \subset(L(G))^{1 / 2}$ then the maximal ideal space of $\mathfrak{M}$ is $G^{\wedge}$; i.e., $\mathfrak{M}$ satisfies (2).

Proof. It follows from the characterization of the radical in Lemma 1, that if $\mathfrak{M}_{2}$ is an $L$-subalgebra of a convolution measure algebra $\mathfrak{M}_{1}$ and if $\mathfrak{R}$ is an $L$-ideal of both $\mathfrak{M}_{1}$ and $\mathfrak{M}_{2}$, then the radical of $\mathfrak{R}$ in $\mathfrak{M}_{2}$ is the intersection with $\mathfrak{M}_{2}$ of the radical of $\mathfrak{N}$ in $\mathfrak{M}_{1}$. Thus, if $L(G) \subset \mathfrak{M} \subset(L(G))^{1 / 2}$, then the radical of $L(G)$ in $\mathfrak{M}$ is $\mathfrak{M}$. It follows that $L(G)$ and $\mathfrak{M}$ have the same maximal ideal space, namely $G^{\wedge}$.

Before considering the converse problem we require a lemma concerning closed semigroups in 1.c.a. groups.

LEMMA 2. If $T$ is a closed proper subsemigroup of the 1.c.a. group $G$ which generates $G$ (i.e., the closed subgroup generated by $T$ is $G$ ), then there is a continuous homomorphism $\gamma$ of $G$ into the additive real numbers $R$ such that $0 \neq \gamma(T) \subset R^{+}$, the set of nonnegative reals.

Proof. If $T$ has an interior point $x$ such that $-x \notin T$, then the lemma follows from results of Rieffel (cf. [4, Theorem 6.4]). We shall prove the lemma directly in the case where $G=K \times R^{n}$ for a compact group $K$, and use this to reduce the general case to Rieffel's result.

We prove the lemma for $G$ of the form $K \times R^{n}$ by induction on $n$. If $n=0$ then $G$ is compact and the lemma holds vacuously, since a closed subsemigroup of a 
compact group must be a group (cf. [7, p. 99]) and, thus, $T$ generates $G$ would imply $T=G$. We now assume the lemma holds for $n=p-1$ and let $G=K \times R^{p}$ for some compact group $K$. Since $T$ is not a group, there is some $x \in T$ for which $-x \notin T$. Since $K$ is compact, $T \cap K$ is a group; hence, $x \notin K$. Note that without loss of generality we may assume that $0 \notin T$ for, if $0 \in T$, we may replace $T$ by $T^{\prime}=x+T$; then $0 \notin T^{\prime}$ and if $\gamma$ is a homomorphism of $G$ into $R$ such that $\gamma\left(T^{\prime}\right) \subset R^{+}$, then $\gamma(T) \subset R^{+}-\gamma(x)$; but $\gamma(T)$ is a semigroup, so $\gamma(T) \subset R^{+}$.

In view of the above considerations, we may, without loss of generality, write $G$ as $H \times R$, where $H=K \times R^{p-1}, x$ is the element $(0,1) \in H \times R$, and $(0, n) \in T$ for positive integers $n$ but not for nonpositive integers $n$.

Let $G^{+}=\{(h, r) \in H \times R: r \geqq 0\}$ and $G^{-}=\{(h, r) \in H \times R: r \leqq 0\}$. If $T \subset G^{+}$then the projection of $H \times R$ on $R$ is the required $\gamma$. If $T \notin G^{+}$we set $T^{-}=T \cap G^{-}$, $Z=\{(0, n): n=0, \pm 1, \pm 2, \ldots\}$, and let $\phi$ be the natural map from $G$ to $G / Z$. Note that $(0,1) \in T$ implies that $\phi\left(T^{-}\right)=\phi\left(T_{0}^{-}\right)$, where

$$
T_{0}^{-}=\{(h, r) \in H \times R:-1 \leqq r \leqq 0\} \cap T^{-},
$$

and since $\phi\left(T_{0}^{-}\right)$is closed, so is $\phi\left(T^{-}\right)$. Also, $Z \cap T^{-}$is empty so $0 \notin \phi\left(T^{-}\right)$and $\phi\left(T^{-}\right)$is a closed proper subsemigroup of $G / Z$. To apply the induction hypothesis we need only show that $\phi\left(T^{-}\right)$generates $G / Z$. If not, then $\phi\left(T^{-}\right) \subset J$ where $J$ is a proper closed subgroup of $G$; if $y \in T$ and $w \in T^{-}$, then $y+w^{n} \in T^{-} \subset \phi^{-1}(J)$ for sufficiently large $n$, and, hence, $y \in \phi^{-1}(J)$; i.e., $T \subset \phi^{-1}(J)$ which contradicts the assumption that $T$ generates $G$. Note that if $p=1$ then $T$ must be contained in $G^{+}$, since $G / Z$ is compact. Thus the lemma is proven in this case.

Now if $p>1$ the noncompact part of $G / Z$ has dimension $p-1 \neq 0$, and we may apply the induction hypothesis and conclude that there is a continuous homomorphism $f$ of $G / Z$ into $R$ such that $0 \neq f\left(\phi\left(T^{-}\right)\right) \subset R^{+}$. We now define a map $g$ of $G$ into $R \times R$ by $g(h, r)=(f(\phi(h, r)), r)$; then $g$ is a continuous homomorphism and $g(T) \cap\{(t, r): t<0, r<0\}$ is empty; i.e., $g(T)$ misses the open third quadrant. From this and the fact that $g(T)$ is a semigroup, it follows readily that the convex hull of $g(T)$ also misses the third quadrant and may thus be separated from it by a straight line $l$ passing through the origin. The line $l$ may contain $g(T)$, in which case we have reduced the problem to the one-dimensional case and may apply the last comment of the previous paragraph. If $g(T) \Phi l$, then there is a continuous homomorphism $\alpha$ of $R \times R$ onto $R$, with kernel $l$, such that $0 \neq \alpha(g(T)) \subset R^{+}$. Then $\alpha$ composed with $g$ is the required map $\gamma$. This completes the induction.

To prove the lemma for $G$ a general l.c.a. group, we use the structure theorem for l.c.a. groups (cf. [5, Theorem 2.4.1]) which says that $G$ contains an open subgroup $G_{1}$ of the form $K \times R^{n}$ for a compact group $K$. Let $\beta$ be the natural map from $G$ to $G / G_{1}$. If $\beta(T)$ is proper in $G / G_{1}$, we may apply the above mentioned result of Rieffel to obtain $\gamma$, since $G / G_{1}$ is discrete. If $\beta(T)=G / G_{1}$ then every coset of $G_{1}$ in $G$ contains an element of $T$. Then $T \cap G_{1}$ must be a proper generating subsemigroup of $G_{1}$ if $T$ is to be a proper generating subsemigroup of $G$. Thus, we 
may apply the result of the previous paragraph to obtain a continuous homomorphism $\gamma_{1}$ of $G_{1}$ into $R$ such that $0 \neq \gamma\left(T \cap G_{1}\right) \subset R^{+}$. We write

$$
G_{1}^{+}=\left\{x \in G_{1}: \gamma_{1}(x) \geqq 0\right\} .
$$

Then $G_{1}^{+}+T$ is proper in $G$, for if $y \in G_{1}$ and $y=x+t$ with $x \in G_{1}^{+}$and $t \in T$, then $y-x \in G_{1} \cap T$ and $\gamma_{1}(y-x)=\gamma_{1}(y)-\gamma_{1}(x) \geqq 0$ so that $y \in G_{1}^{+}$also. Clearly, $G_{1}^{+}+T$ is closed, generates $G$, and has an interior point $x$ such that $-x \notin G_{1}^{+}+T$ (e.g., any $x \in G_{1}$ for which $\gamma_{1}(x)>0$ ). We again apply Rieffel's result and the proof is complete.

Note that Lemma 2 implies that on a semigroup $T$ of the above type there is a proper semicharacter, i.e., a semicharacter $f$ for which $|f| \not \equiv 1$; in fact, $f(x)=e^{-s \gamma(x)}$ for $s>0$ and $x \in T$ is such a semicharacter.

In what follows, $\mathfrak{M}$ will denote a closed subalgebra of $M(G)$ which satisfies (1) and (2). Lemmas 3 and 4 hold for arbitrary l.c.a. groups $G$, while the remainder of the proof of Theorem 2 requires special assumptions on $G$.

LEMmA 3. $\mathfrak{M}$ is weak-* dense in $M(G)$ and $\mathfrak{M}^{\wedge}$ is uniformly dense in $C_{0}\left(G^{\wedge}\right)$.

Proof. Let $T=$ carrier $(\mathfrak{M})$ be the smallest closed subset of $G$ on which each $\mu \in \mathfrak{M}$ is concentrated. Since $\mathfrak{M}$ is an $L$-subalgebra of $M(G), T$ is a closed subsemigroup of $G$, and $\mathfrak{M}$ is weak-* dense in $M(G)$ if and only if $T=G$. Since $\mathfrak{M}$ separates points in $G^{\wedge}, T$ cannot be contained in any closed proper subgroup of $G$; i.e., $T$ generates $G$. If $T \neq G$ we may apply Lemma 2 and obtain a continuous homomorphism $\gamma$ of $G$ into $R$ such that $0 \neq \gamma(T) \subset R^{+}$. Then $\mu \rightarrow \int e^{-\gamma(x)} d \mu(x)$ is a complex homomorphism of $\mathfrak{M}$ which clearly does not correspond to any character of $G$. This contradicts the fact that $\mathfrak{M}$ satisfies (2). We conclude that $T=G$ and $\mathfrak{M}$ is weak-* dense in $M(G)$.

Let $\lambda \in M\left(G^{\wedge}\right)$; then $\lambda^{\wedge}(x)=\int \bar{\chi}(x) d \lambda(x)$ is continuous on $G$, so there exists $\mu \in \mathfrak{M}$ for which $\int \lambda^{\wedge} d \mu=\int \mu^{\wedge} d \lambda \neq 0$. Hence $\mathfrak{M}^{\wedge}$ is a dense subspace of $C_{0}\left(G^{\wedge}\right)$. This completes the proof.

LEMmA 4. If $\mathfrak{M} \cap L(G) \neq(0)$ then $L(G) \subset \mathfrak{M} \subset(L(G))^{1 / 2}$.

Proof. $\mathfrak{M} \cap L(G)$ is an $L$-subalgebra of $L(G)$ and an $L$-ideal of $\mathfrak{M}$. If $\nu \in L(G)$ then Lemma 3 implies that $\nu$ is the weak-* limit in $M(G)$ of a net $\left\{\mu_{\alpha}\right\}$ of elements of $\mathfrak{M}$. If $f \in L_{\infty}(G)$ and $\omega \in \mathfrak{M} \cap L(G)$, then $g(x)=\int f(x+y) d \omega(y) \in C(G)$ and, hence, $\int f d \omega \cdot \mu_{\alpha}$ converges to $\int g d \nu=\int f d \omega \cdot \nu$; i.e., $\left\{\omega \cdot \mu_{\alpha}\right\}$ is a net in $\mathfrak{M} \cap L(G)$ which converges weakly to $\omega \cdot \nu$ and so $\omega \cdot \nu \in \mathfrak{M} \cap L(G)$. It follows that $\mathfrak{M} \cap L(G)$ is an $L$-ideal of $L(G)$. However, the only $L$-ideals of $L(G)$ are $(0)$ and $L(G)$. Hence $L(G) \subset \mathfrak{M}$. Also $\mathfrak{M} \subset(L(G))^{1 / 2}$, otherwise there would be a complex homomorphism of $M(G)$ which was zero on $L(G)$ and nonzero on $\mathfrak{M}$, contradicting the fact that the maximal ideal space of $\mathfrak{M}$ is $G^{\wedge}$.

LEMMA 5. If $G$ contains no copy of $R$, then $L(G) \subset \mathfrak{M} \subset(L(G))^{1 / 2}$. 
Proof. Under this hypothesis, $G$ and $G^{\wedge}$ each contain an open-compact subgroup, by the principal structure theorem (cf. [5, Theorem 2.4.1]). Let $K$ be an opencompact subgroup of $G^{\wedge}$. Since $G^{\wedge}$ is the maximal ideal space of $\mathfrak{M}$, Shilov's theorem (cf. [3, Theorem 3.6.3]) implies the existence of $\mu \in \mathfrak{M}$ such that $\mu^{\wedge}(\chi)=0$ for $\chi \notin K$ and $\mu^{\wedge}(\chi)=1$ for $\chi \in K$. Then $\mu$ is Haar measure on an open-compact subgroup $H$ of $G(H=\{x \in G: \chi(x)=1$ for $x \in K\})$. Thus $\mu \in L(G)$ and the conclusion follows from Lemma 4.

LEMMA 6. If $G$ is of the form $K \times R$ where $K$ is compact, and

$$
G^{+}=\{(k, r) \in K \times R: r>0\},
$$

then there exists a nonnegative measure $\mu \in \mathfrak{M}$ concentrated on $G^{+}$with compact support and $\|\mu\|>1$ such that $\mu^{\wedge}(\chi) \neq 1$ for each $\chi \in G^{\wedge}$.

Proof. It follows from Lemma 3 that it is enough to show the existence of a $\nu \in L(G)$ with the above properties. An example of such a measure $\nu$ is $\nu=2(\rho \times \omega)$, where $\rho$ is Haar measure on $K$ and $\omega$ is Lebesgue measure on $[0,1]$ in $R$.

THEOREM 2. If $G$ contains no copy of $R^{n}$ for $n>1$, then $L(G) \subset \mathfrak{M} \subset(L(G))^{1 / 2}$.

Proof. If $G$ contains no copy of $R$, then Lemma 5 gives us the result; otherwise, the hypothesis, together with the structure theorem for l.c.a. groups, implies that $G$ has an open subgroup $G_{1}$ of the form $K \times R$ with $K$ compact. Let $\mathfrak{M}_{1}$ be the subalgebra of $\mathfrak{M}$ consisting of all measures in $\mathfrak{M}$ which are concentrated on $G_{1}$. It is easily seen that $\mathfrak{M}_{1}$ is an $L$-subalgebra of $M\left(G_{1}\right)$ with maximal ideal space $G_{1}$. Thus the preceding lemmas hold for $\mathfrak{M}_{1}$ as a subalgebra of $M\left(G_{1}\right)$.

Now let $\mu$ be the element of $\mathfrak{M}_{1}$ given by Lemma 6 . Since $\mu^{\wedge}$ never assumes the value one, $\mu$ has an adverse (cf. $[2, \S 21]$ ); i.e., there is an element $\nu \in \mathfrak{M}_{1}$ such that $\mu \nu=\mu+\nu$. If $\mu$ had norm less than one, its adverse would be $\omega=-\sum_{n=1}^{\infty} \mu^{n}$. Since $\mu$ is concentrated on $G_{1}^{+}$, the series $\sum \mu^{n}$ converges on each compact subset of $G_{1}$, but since $\|\mu\|>1$ and $\mu \geqq 0$, it converges to an unbounded measure.

The Laplace-Stieltjes transform, $\tilde{\rho}$, for a measure $\rho$ on $G_{1}=K \times R$ is defined by $\tilde{\rho}(\chi, z)=\int_{K \times R} e^{-z t} \bar{\chi}(k) d \rho(k, t)$, for $\chi \in K^{\wedge}$ and $z$ a complex number, whenever this integral converges. Note that since $\mu$ has compact support, $\tilde{\mu}$ exists and is analytic in $z$ for all $\chi$, and $\mu^{\wedge}(\chi, y)=\tilde{\mu}(\chi, i y)$, for $y$ real, is the Fourier-Stieltjes transform of $\mu$. Since $\nu$ is a bounded measure, $\tilde{\nu}$ exists for $z$ imaginary and

$$
\tilde{\nu}(\chi, i y)=\nu^{\wedge}(\chi, y)=\mu^{\wedge}(\chi, y)\left[\mu^{\wedge}(\chi, y)-1\right]^{-1} .
$$

Also, for sufficiently large positive $r, \int e^{-r t} d \mu(k, t)<1$ and $\int e^{-r t} d \omega(k, t)$ is finite. Thus, for $x \geqq r, \tilde{\omega}(\chi, x+i y)$ exists and equals $\tilde{\mu}(\chi, x+i y)[\tilde{\mu}(\chi, x+i y)-1]^{-1}$.

The function $f(\chi, z)=\tilde{\mu}(\chi, z)[\tilde{\mu}(\chi, z)-1]^{-1}$ is analytic in $z$ except where $\tilde{\mu}(\chi, z)=1$, and at such points it has simple poles. Also, since $\tilde{\mu}(\chi, z)$ approaches zero at infinity for $\operatorname{Re}(z) \geqq 0$, there can be at most finitely many poles of $f$ in the region $\operatorname{Re}(z) \geqq 0$. 
Let $\left\{\rho_{\alpha}\right\}$ be a weak-* approximate identity for $M(G)$ consisting of elements of $L\left(G_{1}\right)$ with compact support and norm one, whose Laplace transforms $\tilde{\rho}_{\alpha}$ have the property that $\tilde{\rho}_{\alpha}(\chi, a+i y)$ is integrable in $(\chi, y)$ for each $a \in R$. Let $g_{\alpha}(k, t)$ be the Radon-Nikodym derivative of $\rho_{\alpha}$ for each $\alpha$; then, the inversion formula (cf. [5], Theorem 1.5.1) implies that

$$
g_{\alpha} * \nu(k, t)=\frac{1}{2 \pi i} \int_{-i \infty}^{i \infty}\left[\sum_{\chi \in K^{\wedge}} e^{z t} \chi(k) f(\chi, z) \hat{\rho_{\alpha}}(\chi, z)\right] d z
$$

and

$$
g_{\alpha} * \omega(k, t)=\frac{1}{2 \pi i} \int_{x-i \infty}^{x+i \infty}\left[\sum_{x \in K^{\wedge}} e^{z t} \chi(k) f(\chi, z) \hat{\rho_{\alpha}}(\chi, z)\right] d z,
$$

for $x \geqq r$. It follows that

$$
g_{\alpha} *(\omega-v)(k, t)=\frac{1}{2 \pi i} \sum_{\chi \in P} \int_{\Gamma} e^{z t} \chi(k) f(\chi, z) \hat{\rho_{\alpha}}(\chi, z) d z
$$

where $P$ is the finite set of points $\chi \in K^{\wedge}$ for which $f(\chi, z)$ has a pole in the region $\operatorname{Re}(z) \geqq 0$, and $\Gamma$ is any simple closed curve in $\operatorname{Re}(z) \geqq 0$ enclosing all such poles. Then $g_{\alpha} *(\omega-\nu)$ converges uniformly on compact subsets of $G_{1}$ to the function

$$
\phi(k, t)=\frac{1}{2 \pi i} \sum_{\chi \in P} \int_{\Gamma} e^{z t} \chi(k) f(\chi, z) d z,
$$

while $\rho_{\alpha} \cdot(\omega-\nu)$ converges weakly, relative to continuous functions with compact support, to the measure $\omega-\nu$. It follows that $\omega-\nu$ is absolutely continuous on each compact set with Radon-Nikodym derivative $\phi$. Thus, if $\lambda$ is the restriction of $\omega-\nu$ to any compact subset of $G_{1}$, we have $\lambda \in L\left(G_{1}\right) \cap \mathfrak{R}_{1} \subset L(G) \cap \mathfrak{M}$. In view of Lemma 4, the proof is complete.

Added in proof. We have recently obtained a proof of Theorem 2 without the condition on $G$. Thus, the conjecture mentioned at the beginning of this paper is true.

\section{BIBLIOGRAPHY}

1. E. Hewitt and K. A. Ross, Abstract harmonic analysis, Springer-Verlag, Berlin, 1963.

2. L. H. Loomis, An introduction to abstract harmonic analysis, Van Nostrand, New York, 1953.

3. C. E. Rickart, General theory of Banach algebras, Van Nostrand, Princeton, N. J., 1960.

4. M. A. Rieffel, A characterization of commutative group algebras and measure algebras, Trans. Amer. Math. Soc. 116 (1965), 32-65.

5. W. Rudin, Fourier analysis on groups, Interscience, New York, 1962.

6. J. L. Taylor, The structure of convolution measure algebras, Trans. Amer. Math. Soc. 119 (1965), 150-166.

7. A. D. Wallace, The structure of topological semigroups, Bull. Amer. Math. Soc. 61 (1955), $95-112$.

UNIVERSITY OF UTAH, Salt Lake City, Utah 